\title{
Last Subject Last Visit Identity
}

National Cancer Institute

\section{Source}

National Cancer Institute. Last Subject Last Visit Identity. NCI Thesaurus. Code C142599.

The identity of the final subject to reach the final scheduled visit, which is a planned or achieved milestone representing the completion of the trial. 